\title{
VARIATIONS OF SHORT PERIODICAL OSCILLATIONS OF EARTH ROTATION
}

\author{
B. Kolaczek \\ Space Research Center, PAS \\ Bartycka 18A, 00-716 Warsaw, Poland
}

ABSTRACT. The short periodical oscillations of variations of Earth rotation parameters (ERP) with periods of about $120,100,80,60,50$ days are the most energetic oscillations after the chandler and seasonal ones. These oscillations were analysed using SLR and VLBI data and the narrow ormsby band-pass and Fourier Transformation filters. Polhodes of these oscillations for the last several years were computed. Amplitudes of these oscillations are of the order of 2-5 mas and are changeable. They are modulated with a period of about 600 days.

\section{INTRODUCTION}

The observational techniques such as satellite laser ranging (SLR), Very Long Baseline Interferometry (VLBI) and Global Positioning system (GPS) have enabled investigations of Earth rotation variations in a wider spectral range. At present even one-day oscillations of the ERP with amplitudes of the order of one miliarcsecond are detectable, but the ERP variations in the entire range of the spectrum are not completly understood. The 120, $80,60,50$ day oscillations reaching maximum amplitudes of the order of 2-5 mas have been detected by many authors, whose paper were reviewed by Dickey (1989a,b, 1991), Dickman (1989), Kolaczek (1991) but their character and their origin are not known well. Some examples of results of analyses of these oscillations by the narrow ormsby band-pass and Fourier Transformation filters are presented in this paper. 
2. ANALYSES OF SHORT PERIODICAL OSCILLATIONS OF THE EARTH ROTATION PARAMETERS AND THEIR RESULTS.

In these analyses two sets of Earth rotations parameters (ERP) were used:

- CSR91.L03 - ERP data for years 1976-1991 determined by the Center for Space Research (CSR) in Austin, Texas, USA from Lageos laser ranging (SLR), (IERS, 1991).

- IRIS91. - 5 day solutions of ERP determined by the National Geodetic Survey (NGS) from VLBI data (IERS,1991).

These data were filtered using Gaussian low pass filter with FWHM=5 days (G5), (Feissel et al.,1984) and the Butterworth high pass filter with cutoff period of 140 or 150 days (B140 or B150), (Otnes and Enochson,1942) in order to get equally spaced data and to remove long periodical variations respectively.

Spectral analyses of such filtered data were carried out by three methods: Maximum Entropy (MESA), Ormsby band-pass filter (Ormsby BPF), (Kosek, 1991) and Fourier Transformation (FT) for confirmation of the results. For example, the FT spectra of CSR91.L03 x pole coordinate variations in years 1980-1990 and of IRIS91 UT1-UTC variations in years 1983-1990 are given in Figs. 1, 2.

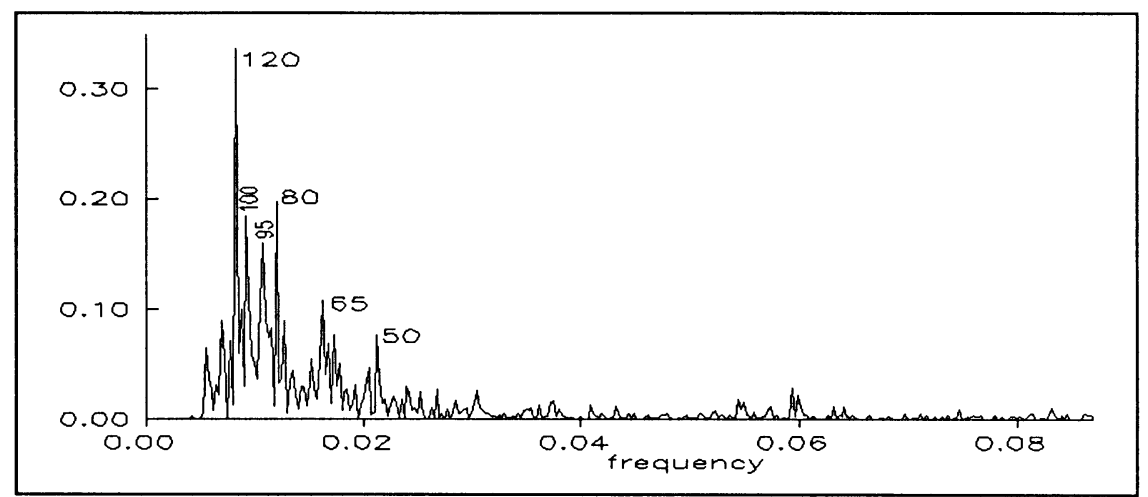

Fig.1. The FT spectra of filtered residuals of $x$ CSR91.L03 pole coordinate variations in years 1978-1991.

The most energetic oscillations of CSR91.L03 pole coordinates and IRIS91 UT1-UTC were filtered using the Ormsby (Ormsby, 1961) and FT filters. The FT filter is 
a difference between the input data and data obtained by inverse FT with a chosen frequency band removed.

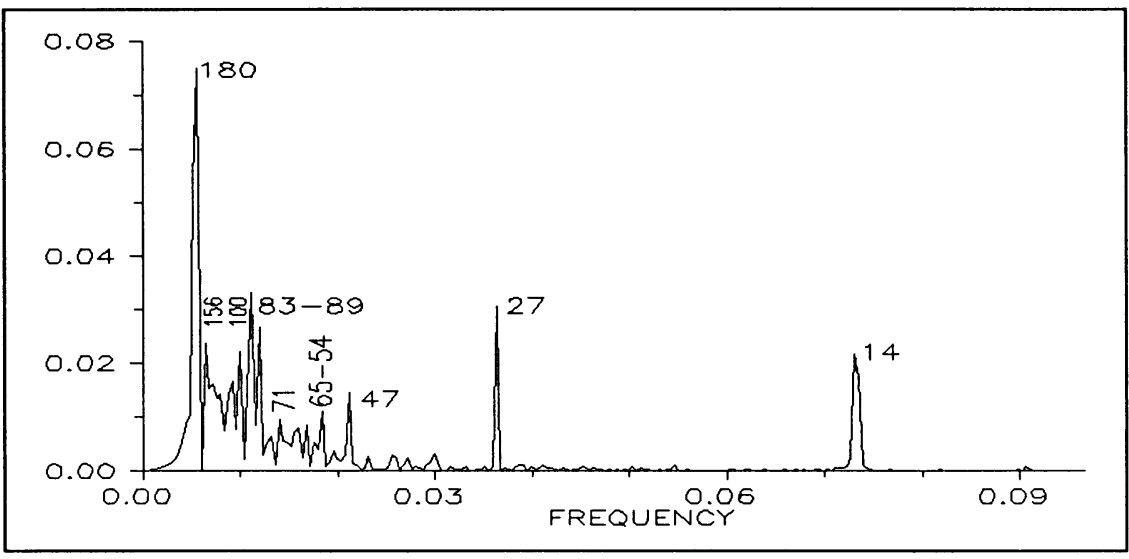

Fig.2. The FT spectrum of filtered residuals of IRIS91 UT1-UTC variations in 1983-1990.

The most energetic oscillations in pole coordinate variations with periods of about 120 and 100 days were analysed earlier (Kolaczek, 1992). These oscillations are eliptical ones with changeable amplitudes, which reach a maximum of 4 mas. Their beating oscillations have similar amplitude variations, which are modulated with a period of about 1.5-2.0 years (Kolaczek, 1992). The oscillations of pole coordinates with periods of about $80,60,50$ days have a similar character. In the last case amplitudes are a little smaller. For example, polhodes of 120 and 60 day oscillations of the CSR91.L03 pole motion filtered by the FT filter in the chosen time interval are shown in Fig.3,4. The beating oscillations in the bands of periods of about $80,60,50$ days have also modulated amplitudes. The MESA of envelopes of amplitude variations determined periods of these modulations of the order of 600 days. Similar results were obtained for the 120-day oscillations of polar motion using the narrow ormsby band-pass filter.

The most energetic oscillations of IRIS91 UT1-UTC variations in the part of the spectrum considered here were filtered using the FT filter. They are shown in Fig.5. They have changeable amplitudes reaching maxima of 1-1.5 ms. Elimination of the filtered oscillations with periods of 130, 110, 90, 50 days from the original residuals of UT1-UTC obtained after filtering with the 
G5-B140 band pass filter mentioned at the begining gives final residual smaller than $2 \mathrm{~ms}$ (Fig. 6).

The results show a similar character to periodical oscillations of polar motion in the part of the spectrum considered here and indicate a possibility for future investigations.
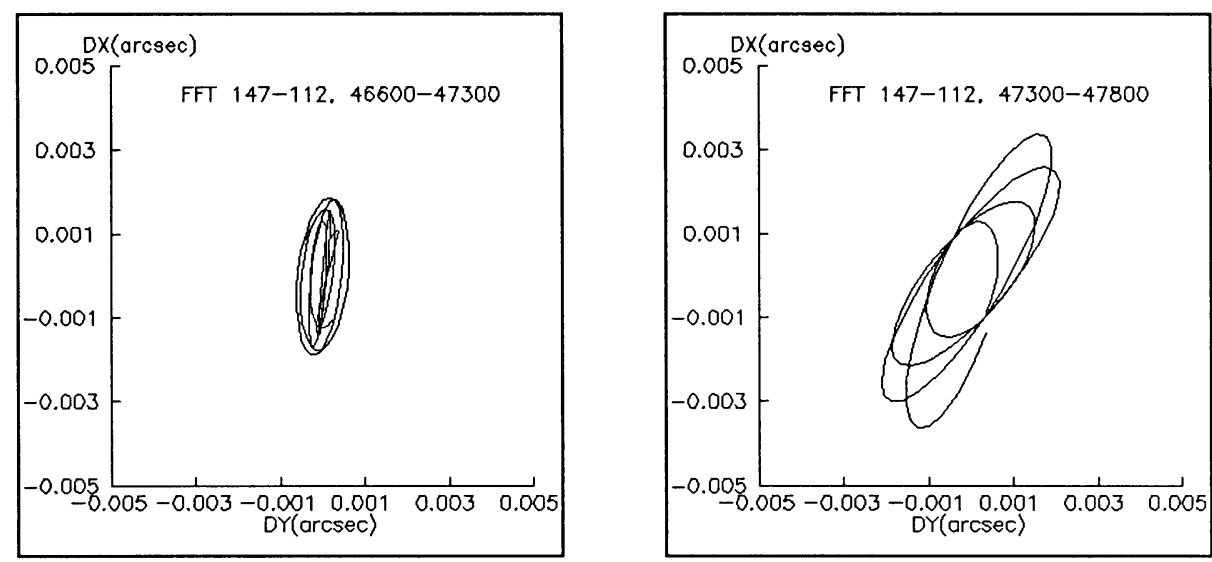

Fig. 3. Polhodes of the 120 day oscillation of CSR91.L03 pole motion filtered by the FT filter.
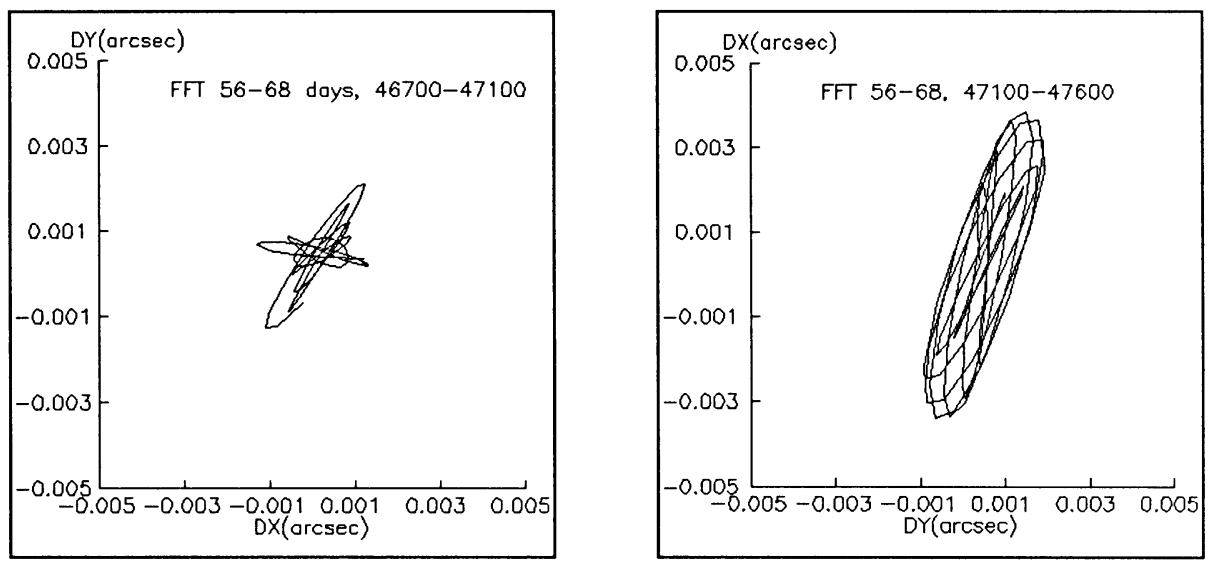

Fig.4. Polhodes of the 60 day oscillation of CSR91.L03 pole motion filtered by the FT filter. 


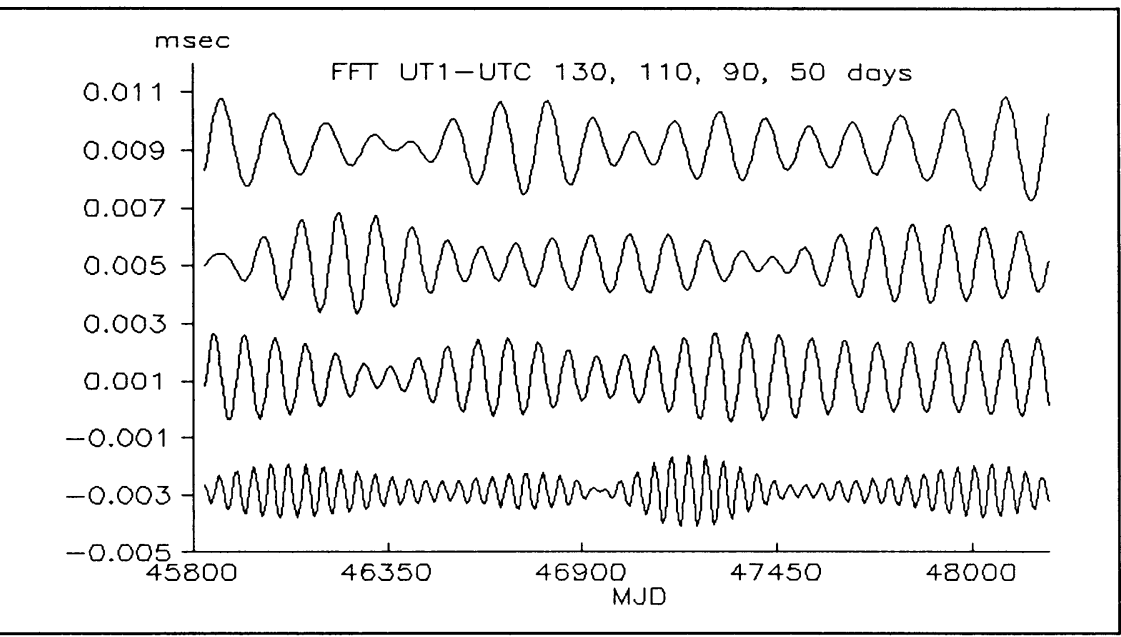

Fig.5. Short periodical oscillations with periods 130 , 110, 90, days of IRIS UT1 - UTC variations filtered by the FT filter.

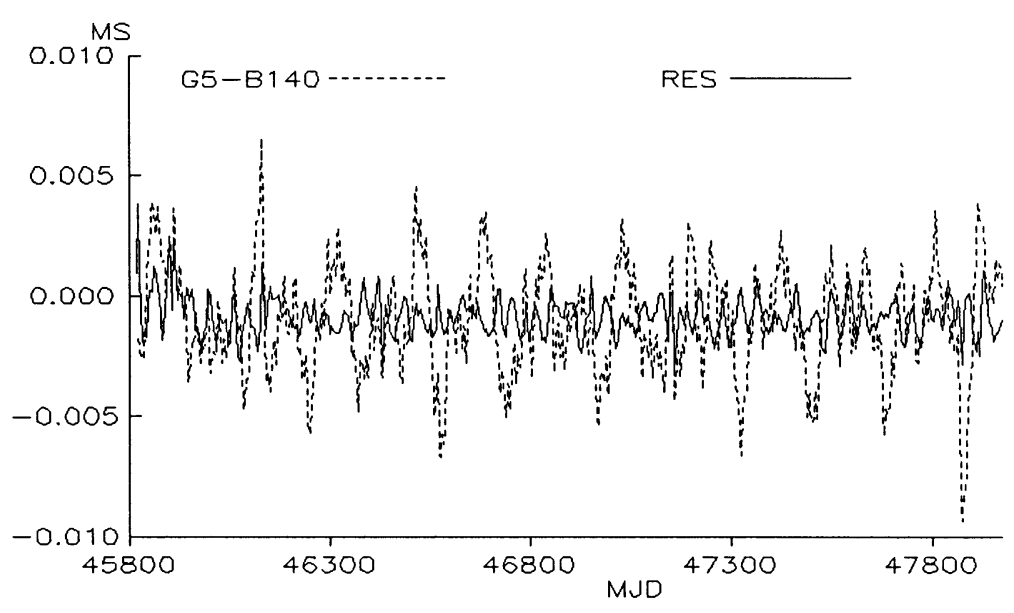

Fig. 6. Comparison of residuals of IRIS91 UT1 - UTC variations filtered using the G5-B150 band pass filter with residuals obtained after elimination of oscillations with periods of $130,110,90,50$ days. 
ACKNOWLEDGEMENT. The researches were supported by the U.S. -Poland Maria Sklodowska-Curie Joint Fund II under the project No PAN/SMI-92-97.

\section{REFERENCES}

Dickey J.O., 1989a, "Axial Rate of Spin of the Earth", Proc. of the Int. Conference on The Interdisciplinary Role of Space Geodesy, Erice, Italy, Mueller,1.1. Zebrini eds., Springer Verlag, New York.

Dickey J.O., Herring T.A., O'Connel R.J., Smylie D.E., 1989b, "Short-Term Dynamics of the Solid Earth", Proc. of Int. Conference on the Interdisciplinary Role of Space Geodesy, Erice, Italy, Mueller I.I., Zerbini eds., Springer Verlag, New York.

Dickey J.O., 1991, Final Report of Special Study Group 5 98 Atmospheric Excitation of the Earth Rotation, XX IUGG General Assembly, Vienna, 1991.

Dickman S.R., 1989, "Polar Motion", Proc. of Int. Conference on the Interdisciplinary Role of space Geodesy, Erice, Italy, Mueller I.I., Zerbini eds., Springer Verlag, New York.

Feissel M. and Lewandowski W.,1984, "A comparative Analysis of Vondrak and Gaussian Smoothing Techniques", Bull. Geod. 58, 464-474.

IERS, 1989 - 1991 IERS Annual Reports 1980 and 1991, Paris observatory, Paris, France.

Kolaczek B., Brzeziński A., Kosek W., Nastula J., Hozakowski W., Soloducha B., 1991 Investigations of Earth Rotation Variations, Artificial Satellites, Planetary Geodesy - No 15, Warsaw, Poland.

Kolaczek B., 1992, "Variations of Short Periodical Oscillations of Earth Rotation with periods of ranging from 10-140 days", Report No.419 Dept. of Geodetic and Surveying, OSU, Columbus, Ohio USA

Kosek W., 1991. On high Frequency Spectral Analysis of Very Noisy Stochastic Processes and its Application to the ERP series determine by VLBI and SLR, U.S. Naval Observatory Circular No.177, Washington DC 20392-5100, Feb 15.

orsmby J.F.A., 1961, Design of Numerical Filters with Application to Missile Data Processing, J. Assoc. Comput. Mach. 8, 440-466.

otnes and Enochson L. 1972, Spectral Analyses and Time Series, Academic Press. London. 